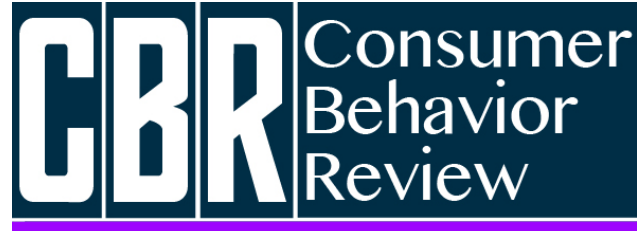

Revista Comportamento do Consumidor
Trindade, J. R., Vasconcelos Júnior, R. M., Maciel Neto, T., \& Aguiar, E. C. (2020). Crowding e Perfil do Consumidor: Influência na imagem varejista e intenção de compra do consumidor jovem. Consumer Behavior Review, 4(3), 309-323.
ISSN: 2526-7884

Editor: Prof. Dr. Marconi Freitas da Costa Journal's e-mail: cbr@ufpe.br
Evaluation: Double blind review

Received: May 23, 2020

Accepted: August 27, 2020

\title{
CROWDING E PERFIL DO CONSUMIDOR: INFLUÊNCIA NA IMAGEM VAREJISTA E INTENÇÃO DE COMPRA DO CONSUMIDOR JOVEM
}

Crowding and Consumer Profile: Influence on the retailer image and intent to purchase the young consumer

ORCID: https://orcid.org/0000-0003-1538-4521

E-mail: rtjaiany@gmail.com

Renato Meira Vasconcelos Júnior ${ }^{1}$

ORCID: https://orcid.org/0000-0002-1174-1395

E-mail: renatojuniorm@gmail.com

Temístocles Maciel Neto ${ }^{1}$

ORCID: https://orcid.org/0000-0001-8812-9332

E-mail: teminetotmn@gmail.com

Edvan Cruz Aguiar ${ }^{1}$

ORCID: https://orcid.org/0000-0002-3433-6210

E-mail: edvan.ed@gmail.com

${ }^{1}$ Universidade Federal de Campina Grande, Campina Grande, Brazil

\section{Resumo}

0 estudo investigou como a densidade humana e o perfil dos outros consumidores influenciam a imagem do varejista e as intenções de compra de consumidores jovens. Assumindo

\begin{abstract}
The study investigated how human density and the profile of other consumers influence the retailer's image and the purchase intentions of young consumers. Assuming an
\end{abstract}


uma abordagem quantitativa de caráter experimental, o estudo foi realizado junto a 131 jovens consumidores. A partir dos resultados encontrados, evidencia-se que a densidade humana pode influenciar a imagem de loja, entretanto, ela sozinha não influencia a decisão de compra. Quanto ao perfil dos outros consumidores, esse não exerce efeito sob a imagem de loja e sob a intenção de compra, sugerindo que o público jovem tende a ser menos impactado pela presença de outros consumidores. 0 trabalho contribui para o entendimento do fenômeno de crowding e da similaridade percebida em ambientes varejistas, trazendo evidências sobre consumidores jovens.

Palavras-chave: Crowding, similaridade percebida, público jovem, varejo. experimental quantitative approach, the study was carried out with 131 young consumers. From the results found, it is evident that human density can influence the store image, however, it alone does not influence the purchase decision. As for the profile of other consumers, this has no effect on the image of the store and on the intention to buy, suggesting that the young audience tends to be less impacted by the presence of other consumers. The work contributes to the understanding of the crowding phenomenon and the similarity perceived in retail environments, bringing evidence about young consumers.

Keywords: Crowding, perceived similarity, young people, retail.

\section{INTRODUÇÃO}

O varejo é considerado uma das principais fontes econômicas no mundo. No Brasil, essa realidade não poderia ser diferente, dado a sua grande importância no cenário nacional. Toda essa conjuntura nesse ambiente tem gerado uma série de desafios gerenciais e operacionais. Segundo Santos e Santos (2019), essa dinâmica exige uma estratégia integrada que envolve todos os processos tanto no ambiente interno como externo da empresa. Essas, necessitam estar em constante evolução e amadurecimento, apresentar um diferencial, desde o produto físico até o consumo final, em todas as atividades que compõem as necessidades e experiências do consumidor durante todo o processo de compra.

O ramo varejista desempenha um papel indispensável no que tange a compreensão dos consumidores, por serem grandes locais de aquisição de bens e serviços, ambientes como esses geram estímulos emocionais, podendo influenciar na satisfação e nas experiências de consumo das pessoas. No que se refere às atitudes e comportamentos de compra, em ambientes de varejo, estudos evidenciaram que a presença social pode atuar como moderadora. Fatores como a presença e o comportamento de outros consumidores, possuem um grande impacto na medida que influenciam diretamente na percepção dos indivíduos quanto a qualidade dos serviços (Baker, 1986; Brocato, Voorhees, \& Baker, 2012; Aguiar \& Farias, 2015; Santos \& Santos, 2019; Aguiar \& Farias, 2020).

Ainda sobre os aspectos dos centros varejistas, quanto aos fatores sociais, o estudo sobre a presença de outros consumidores no mesmo ambiente de varejo levaram a análises acerca da densidade humana e como ela se relaciona com a experiência vivida (Stokols, 1972; Eroglu \& Harrel, 1986; Hui \& Bateson, 1991). Através dessas análises, o conceito multidimensional composto pela percepção de densidade humana e espacial em um dado ambiente varejista, o fenômeno conhecido como crowding foi explorado.

Para Aguiar e Farias (2020), o fato de um indivíduo estar compartilhando o mesmo ambiente com muitos outros consumidores, faz com que este consumidor alvo possa ter respostas emocionais e comportamentais negativas como, permanecer menos tempo naquele ambiente e consequentemente comprar menos. 0 perfil do consumidor é outro entre os fatores sociais que em contato com o de outros consumidores, despertaram estudos de similaridade percebida. Estudos sugerem que a similaridade percebida com outros compradores afeta direta e indiretamente a satisfação dos clientes, ou seja, algumas características observáveis (idade, gênero, aparência, gênero, etc.) podem influenciar 
a maneira como os consumidores avaliam sua experiência de compra (Aguiar \& Farias, 2015; Kwon, Ha \& Im, 2016).

A Teoria do Impacto Social (SIT) diz que o consumidor com a intenção de compra sente-se mais confortável mediante a presença de outros compradores com características parecidas com as dele nesse momento (Latané, 1981). Nesta perspectiva, sobre os consumidores mais jovens, Thakor, Suri e Saleh (2008), identificaram alterações nas avaliações quanto ao serviço e as intenções de compra quando na presença de consumidores mais velhos (meia-idade e/ou idosos).

Dessa forma, o objetivo do presente estudo foi verificar de que maneira a densidade humana e o perfil dos outros consumidores influenciam a imagem do varejista e as intenções de compra do consumidor jovem. $\mathrm{O}$ conhecimento destas variáveis pode trazer evidências sobre o comportamento dos consumidores desse segmento, visto que, existem poucos estudos que focam nesse grupo.

\section{REFERENCIAL TEÓRICO}

\section{Dimensão social do ambiente varejista}

O ambiente varejista possui um papel imprescindível para a compreensão das necessidades dos consumidores. 0 que se explica por ele ser o principal elo para oferecer acesso à população, bem como ser o setor que melhor se adapta às necessidades de cada localidade (Barki, Botelho, \& Parente, 2013). O varejo é uma das principais fontes econômicas do Brasil, dispondo de um alto índice de empregabilidade, visto que mais de $40 \%$ das empresas brasileiras desempenham atividades no setor e o grande número de players favorecem a sua constante evolução e amadurecimento (Santos \& Santos, 2019).

Ainda segundo os autores, o avanço tecnológico tem proporcionado a esse ambiente a criação de valor, que em conjunto com o preço são seus principais diferenciais competitivos, proporcionando novas estratégias como a servitização (produtos tangíveis + serviços) ou PSS (Product Service System). Sendo essas o grande diferencial que envolve além do produto físico, uma série de atividades intangíveis que compõem a necessidade do consumidor não somente pelo produto em si, mas também pelas experiências durante todo o processo desde a compra até o consumo final.

0 ambiente de venda de uma loja é um fator estratégico de suma importância no que concerne a satisfação do consumidor. Este provoca estímulos, atitudes, além de uma carga emocional e de informação, auxiliando-o a fazer uma escolha e convencendo-o a realizar a compra (Aguiar, Farias, Gomes, \& Gomes, 2015). Segundo Baker (1986) as pessoas formam opiniões sobre a realidade a partir das aparências, enquanto que a percepção é influenciada pela tangibilidade, criando expectativas sobre os serviços que perpassam o contato físico real com o produto que estão adquirindo.

No que se refere às atitudes e comportamentos de compra em ambientes de varejo, estudos evidenciaram que a presença social pode atuar como agente moderador, tanto no que diz respeito aos clientes, como das pessoas detentoras de serviços do varejo. Além disso, a presença e o comportamento de outros consumidores, possuem um grande impacto na medida que influenciam diretamente na percepção dos indivíduos quanto a qualidade dos serviços, bem como podem interferir nas reações individuais do consumidor, sejam elas comportamentais, afetivas ou atitudinais (Baker, 1986; Brocato et al., 2012; Aguiar \& Farias, 2015; Aguiar \& Farias, 2020).

Os indivíduos podem influenciar uns aos outros, a partir de estímulos relacionados ao número e proximidade das pessoas em um espaço físico. A Teoria do Impacto Social (SIT), revela o efeito das pessoas sobre um indivíduo, que pode ser de diferentes maneiras. Ele é resultado de diferentes "forças sociais", que podem ser: tamanho (quantidade numérica de pessoas), proximidade física entre as pessoas (imediatismo) e a intensidade (o quanto o indivíduo considera importante a presença social) (Latané, 1981; Aguiar \& Farias, 2015).

O consumo está interligado diretamente ao desejo das pessoas em impor sua personalidade ou individualidade, sendo um fator que leva à realização pessoal. No entanto, as rápidas mudanças advindas da socialização, ocasionaram o encurtamento das relações sociais. Essas tornaram-se decisivas, influenciando diretamente desde o estilo vida das pessoas, até a forma de consumir, deixando os consumidores mais voláteis em seu comportamento de compra (Santos \& Santos, 2019). Nessa perspectiva, destaca-se a dimensão social do ambiente varejista, isto é, quando as pessoas que 
compartilham o ambiente com outros consumidores exercem influências frente às suas respostas emocionais e comportamentais, ou seja, na sua experiência de compra (Aguiar et al., 2015).

\section{Percepção de Crowding e similaridade percebida}

É de fundamental importância para continuidade de uma organização ser adaptável ao ambiente, perceber os sinais ambientais e tê-los como referenciais para o desenvolvimento de suas atitudes. Este é um dos principais fatores para compreender e complementar uma organização. É evidente que toda empresa depende de seu ambiente, e cada uma adota estratégias internas para aprender a responder de maneira diferenciada às pressões ambientais percebidas (Albuquerque, 2007).

Os estudos de crowding buscam compreender melhor como os consumidores se comportam frente a diferentes níveis de densidade e espaciais num ambiente de lojas, sendo essas suas principais dimensões. Esse fenômeno influencia diretamente os consumidores no que se refere às suas emoções e percepções. Assim, à medida que há variação no estado emocional dos consumidores devido à maior ou menor percepção de crowding, existirão diferenças nas respostas comportamentais e avaliações da experiência de compra (Eroglu \& Harrell, 1986; Brandão, 2012; Aguiar et al., 2015; Aguiar \& Farias, 2020).

Para Stokols (1972), diferente de densidade, percepção de crowding é um estado psicológico e experiencial verificado quando a busca por espaço de uma pessoa excede a realidade do local em que ela se encontra. Embora essas estejam conectadas, condições de alta densidade são necessárias, mas não suficientes para que ocorra crowding. Existe uma grande distinção entre as duas dimensões e ambas têm servido como ponto principal para pesquisadores analisarem as reações emocionais e comportamentais, envolvendo ainda uma sobrecarga de estímulos sociais ou restrição física. A perda de controle social e privacidade aumenta a experiência de crowding, assim a inabilidade ou habilidade para controle das interações e a redução da privacidade são consideradas determinantes interpessoais da experiência de crowding (Stokols, 1972; Brandão, 2012).

Ressalta-se a importância de estudar esse fenômeno, visto que ele influencia diretamente nas respostas do consumidor (emocionais, atitudinais e comportamentais) e na sua avaliação global do ambiente varejista (Aguiar et al., 2015). Além disso, grande parte desses estudos ressaltam as experiências negativas quanto ao comportamento de compra e satisfação dos consumidores motivados pelo incremento da densidade, provocando menor permanência na loja, menor satisfação, entre outros (Eroglu \& Harrell, 1986; Hui \& Bateson, 1991; Machleit, Eroglu, \& Mantel, 2000; Eroglu, Machleit, \& Barr, 2005). Porém, outros estudos revelam situações em que a alta densidade humana, nem sempre provocam reações negativas do consumidor, no que tange a sua experiência de compra (Lee, Kim, \& Li, 2011).

Algumas características observáveis (idade, gênero, aparência, gênero, etc.) em conjunto com a contiguidade, o volume e a importância da presença social, podem influenciar a maneira como os consumidores avaliam sua experiência de compra (Aguiar \& Farias, 2015). Estudos de Thakor et al. (2008) identificaram alterações nas avaliações quanto ao serviço e as intenções de compra quando consumidores mais jovens perceberam a presença de consumidores mais velhos (meia-idade e/ou idosos).

Segundo Aguiar e Farias (2015), o impacto dos outros consumidores sob as avaliações individuais com relação ao varejista, vem sido reconhecido especialmente quando são identificadas (dis)similaridades entre o consumidor e os demais presentes no ambiente. Os efeitos positivos da similaridade podem também ser compreendidos de modo que os indivíduos similares terão atração interpessoal. Por causa dessa atração os indivíduos experimentarão resultados positivos. Assim, mesmo em condições contrárias, às pessoas tendem buscar à solução dos conflitos, mas quando se percebem como dissimilares, propende ao afastamento e ao confronto (Silveira \& Hanashiro, 2009).

Sendo assim, para Brocato, Voohrees e Baker (2012), similaridade é o quanto o consumidor se identifica com as demais pessoas presentes no ambiente. Portanto, além da análise física do ambiente e da experiência de crowding, a percepção quanto aos outros consumidores exerce uma forte influência nas respostas do consumidor, demonstrando o impacto da similaridade percebida nas 
respostas emocionais, atitudinais e comportamentais das pessoas (Uhrich \& Benkenstein, 2012; Raajpoot, Jackson, \& Lefebvre, 2013; Aguiar \& Farias, 2015; Aguiar \& Farias, 2020).

\section{Hipóteses}

Nesse sentido, estudos sobre crowding revelam efeitos positivos e negativos no comportamento do consumidor quanto à presença de aglomeração de consumidores em ambiente de loja (Lee et al., 2011), levando-o a conclusões sobre a imagem de loja. Neste ponto, apresenta-se a primeira e a segunda hipótese de estudo:

H1a - Densidade alta (baixa) geram uma imagem negativa (positiva) da loja;

H1b - Densidade alta (baixa) gera um menor (maior) intenção de compra.

De outra maneira, outro fator que exerce forte influência quanto a experiência do consumidor, é a similaridade percebida, levando em consideração sua inferência numa série de respostas individuais, como: imagem de loja, lealdade, satisfação, entre outros (Aguiar, 2016). Aqui, são apresentadas as seguintes hipóteses:

H2a - Existe diferença na imagem de loja conforme o perfil de outros consumidores;

H2b - Existe diferença nas intenções de compra do consumidor conforme o perfil dos outros consumidores.

Concluída a apresentação da revisão de pesquisas teórico-empíricas e as hipóteses propostas, será apresentada a seção método da pesquisa.

\section{MÉTODO DE PESQUISA}

\section{Natureza da pesquisa}

Para atender o objetivo de pesquisa a investigação assume uma natureza de caráter experimental, no qual se caracteriza como um tipo de pesquisa com a proposta de evidenciar um relacionamento entre causa e efeito entre as variáveis e propor interpretações dos resultados (Gil, 2002). Ainda segundo o autor, para que uma pesquisa seja experimental é necessário que suas variáveis sejam intencionalmente manipuladas e apoiadas com suporte teórico, permitindo a formulação de hipóteses que são verificadas empiricamente por meio de coleta e análise dos dados. Dessa forma, para a pesquisa ser considerada efetivamente de natureza experimental, se manipulou as variáveis independentes, usando desenhos experimentais do tipo fatorial para mensurar o efeito dessas variáveis interagindo entre si e em diferentes níveis.

Para relação de causa e efeito deste estudo, utilizou-se como variáveis independentes a densidade humana e o perfil dos outros consumidores presentes em um ambiente varejista, considerando cada um deles com dois níveis. Como variáveis dependentes estabeleceu-se: imagem de loja e intenção de compra. As pesquisas de caráter fatorial também são conhecidas pelo termo between subjects (Wickens \& Keppel, 2004) em que cada um dos grupos experimentais de estudo é submetido apenas um tratamento, ou seja, na execução do experimento os sujeitos da pesquisa são aleatoriamente distribuídos pelas condições previamente estabelecidas.

A pesquisa corresponde a um experimento de desenho fatorial, between subject, 2 (densidade humana) X 2 (perfil dos outros consumidores). 0 Quadro 1 apresenta o desenho do experimento.

\begin{tabular}{|c|c|c|}
\cline { 2 - 3 } \multicolumn{1}{c|}{} & Densidade Alta & Densidade Baixa \\
\hline Perfil A & Perfil A, Densidade Alta & Perfil A, Densidade Baixa \\
\hline Perfil B & Perfil B, Densidade Alta & Perfil B, Densidade Baixa \\
\hline
\end{tabular}

Fonte: Elaboração dos autores.

Quadro 1: Desenho do experimento fatorial 2x2. 
Com isso, verifica-se a existência de 4 condições a partir da combinação de dois níveis de densidade humana e os dois perfis dos outros consumidores na situação onde os sujeitos de pesquisa foram estabelecidos. Foi aplicada a técnica de dados intergrupo (Hair, Black, Babin, Anderson, \& Tatham, 2009; Malhotra, 2012) em que cada sujeito da pesquisa é exposto a apenas uma das condições do experimento. Tal medida é justificada pelo fato do membro ter sua resposta influenciada em função da situação anterior, podendo gerar um viés nas suas impressões quanto às combinações da fatorial.

\section{Procedimento de coleta \\ Delineamento dos Cenários de pesquisa}

Como cenário de pesquisa foi determinado o uso de uma loja de roupas, uma vez que esse ambiente varejista tradicional amplia a compreensão do papel da dimensão social no comportamento consumidor em contextos de consumo diferentes. A literatura também evidencia que estudos relacionados à dimensão social são mostrados em contexto de serviços, como também em ambientes de loja. Os consumidores tendem a reforçar sua imagem de loja também pela percepção da presença social (outros consumidores). Dessa maneira, estudos relacionados à similaridade percebida sobre esses ambientes também são melhores explorados.

Após a escolha do Cenário, foram estabelecidas as condições do experimento (between subject). Foram convidados figurantes para participar da construção do contexto de compra na loja, por meio de uma sessão fotográfica. 0 objetivo foi de reproduzir as situações a partir da combinação dos dois fatores (variáveis independentes) e seus respectivos níveis, correspondendo às quatro condições possíveis.

Na sessão fotográfica, se percebeu que se fazia necessário a presença de 10 figurantes, entre homens e mulheres, para se chegar às duas situações de densidade humana (baixa e alta). Com relação ao perfil dos consumidores, a vestimenta dos figurantes era pista social que permitia inferir aos sujeitos de pesquisa suas percepções sobre similaridade percebida.

Foram definidos dois perfis de clientes de loja, no qual um representava através da vestimenta um consumidor de baixa renda e outro consumidor de classe alta com base em um senso comum. Devido a isso, as duas condições do "Perfil dos Outros Consumidores" tiveram de ser validadas posteriormente.

\section{Amostragem}

Os estudos de caráter experimental, demonstram que é necessário no mínimo 30 participantes por condição, de acordo com Malhotra (2012). Em virtude disso, considerando as quatro condições diante da combinação de duas variáveis independentes e considerando que cada um deve ter dois níveis, o tamanho mínimo de respondentes é de 120.

\begin{tabular}{|c|c|}
\hline Grupo de Sujeitos & Condição do Experimento \\
\hline $\begin{array}{c}\text { Grupo 1 (Mínimo 30 } \\
\text { respondentes) }\end{array}$ & Perfil A, Densidade Alta \\
\hline $\begin{array}{c}\text { Grupo 2 (Mínimo 30 } \\
\text { respondentes) }\end{array}$ & Perfil A, Densidade Baixa \\
\hline $\begin{array}{c}\text { Grupo 3 (Mínimo 30 } \\
\text { respondentes) }\end{array}$ & Perfil B, Densidade Alta \\
\hline $\begin{array}{c}\text { Grupo 4 (Mínimo 30 } \\
\text { respondentes) }\end{array}$ & Perfil B, Densidade Baixa \\
\hline
\end{tabular}

Fonte: Elaboração dos autores.

Quadro 2: Distribuição da amostra por condições do Experimento.

Os sujeitos da pesquisa foram estudantes de cursos de graduação, de uma instituição de ensino superior localizado no interior do nordeste brasileiro. A amostra foi do tipo não probabilística e por 
acessibilidade. Foram enviados, aleatoriamente, e-mails para os discentes regularmente matriculados no curso de Administração (sistema controle acadêmico do curso fornecido pela coordenação), e discentes de outros cursos. A coleta aconteceu entre os meses de maio a junho de 2019. O Quadro 2 representa a distribuição do tamanho total da amostra por células (condições do experimento).

\section{Instrumento e procedimento de coleta}

Para instrumento de coleta, foi elaborado um survey através de um questionário online contendo 33 questões estruturado em duas partes. A primeira parte se refere aos construtos teóricos da Similaridade percebida (Byrne, 1971; Sirgy et al., 1997; Brocato et al., 2012), Percepção de crowding (Eroglu \& Machleit, 1990; Machleit, Kellaris, \& Eroglu, 1994); aos construtos atitudinais, imagem de loja (Golden, Albaum, \& Zimmer, 1987; Grewal, Baker, Levy, \& Voss, 2003), e percepção de preço (Srivastava \& Lurie, 2004; Kukar-Kinney \& Grewal, 2007, Kukar-Kinney, Walters, \& Mackenzie, 2007); aos construtos comportamentais, intenção de compra (Mehrabian \& Russell, 1974; Grewal et al., 2003; Rompay, Galetzka, Pruyn, \& Garcia, 2008), comportamento de aproximação e afastamento (Mehrabian \& Russell, 1974; Grewal et al., 2003), necessidade de toque (Peck \& Childers, 2003); por fim, a tolerância ao crowding (Machleit et al., 2000). A segunda parte se refere a questões descritivas (sexo, idade, renda, área do curso).

Foi utilizado uma escala do tipo Likert de 5 pontos, variando de (1) discordo totalmente a (5) concordo totalmente. Como se tratam de variáveis latentes, os níveis de densidade humana e perfil dos outros consumidores não correspondem às escalas de mensuração.

\section{Procedimentos de Análise}

Para procedimento de análise, se fez necessário uma inspeção na base de dados (missing data) e a verificação de valores excessivamente reduzidos e elevados (outliers). Sobre os outliers, estes foram identificados a partir das magnitudes de seus escores padronizados, sendo valores de observações superiores a 3 ou inferiores a -3 retirados da amostra.

A confiabilidade composta e a variância média extraída, também corresponderam aos procedimentos que objetivaram conferir a validade das escalas escolhidas para mensurar os construtos de interesse desta pesquisa (variáveis latentes). Segundo Hair, Black, Babin, Anderson e Tatham (2009), o valor mínimo aceitável para a confiabilidade composta é 0,70. A variância média extraída (AVE) corresponde a uma medida indicativa de confiabilidade do modelo dos construtos. 0 valor mínimo considerado para a AVE é 0,50 (Hair et al., 2009). Ela é uma medida que com base na confiabilidade dos construtos, sinaliza a confiabilidade do modelo.

\section{ANÁLISE E DISCUSSÃO DOS RESULTADOS}

Nesta etapa são demonstrados os resultados encontrados na pesquisa a partir dos tratamentos estatísticos utilizados junto aos dados obtidos pelos sujeitos da pesquisa.

\section{Caracterização da amostra}

A pesquisa obteve uma amostra de 131 respondentes, sendo que $54,12 \%$ do sexo feminino, $45,03 \%$ do sexo masculino e $0,75 \%$ denominados como outros. Com relação a renda familiar dos respondentes, $61,06 \%$ possuem renda familiar de até 2 salários mínimos, 21,37\% até 2 a 4 salários mínimos, 12,21 \% até 4 a 10 salários mínimos e 5,34 \% possuem até 10 salários mínimos. As áreas de graduação que os sujeitos cursam, 60,30 \% são de humanas, $28,24 \%$ são de exatas e $11,45 \%$ são de biológicas. A idade média dos respondentes é de 21 anos.

Considerando o lócus de pesquisa se tratar de Cenários diferentes, o perfil da amostra encontra-se dentro do perfil pré-definido pelos pesquisadores e consequentemente, ao propósito do estudo. 


\section{Análise da distribuição da amostra e validação das escalas}

Foram utilizados a Análise Fatorial Exploratória (AFE) e o Alfa de Cronbach, estas técnicas estatísticas são conduzidas para conferir a dimensionalidade e a confiabilidade das variáveis (construtos) (Hair et al., 2009).

Com relação à AFE, o método de rotação ortogonal das variáveis, VARIMAX, permitiu a identificação dos fatores (variáveis latentes). 0 KMO foi de 0,88 e o Teste de Esfericidade de Bartlett 1132,019, com 78 graus de liberdade a um nível de significância de 0,001. 0 passo seguinte permite averiguar a consistência interna dos construtos.

Tabela 1

Coeficientes Alpha dos construtos.

\begin{tabular}{c|c}
\hline Construtos & Cronbach's Alpha (coeficiente) \\
\hline Similaridade Percebida & 0,95 \\
\hline Percepção de Crowding & 0,88 \\
\hline Intenção de compra & 0,93 \\
\hline Imagem de loja & 0,90 \\
\hline
\end{tabular}

Fonte: Elaboração dos autores.

O Cronbach's Alpha é um coeficiente que varia de 0 a 1 , sendo que, acima de 0,6 pode-se considerar satisfatória a confiabilidade da escala (Malhotra, 2012). Conforme a tabela 1, pode-se garantir a validade interna da escala, visto que os testes apresentaram resultados acima de 0,6 para todos os quatro construtos, permitindo a continuidade das análises.

\section{Mensuração dos construtos}

Foi verificado as médias das respostas dos sujeitos de pesquisa relativas aos construtos de similaridade percebida (SP), percepção de crowding, intenção de compra (IC) e imagem de loja (IL).

Tabela 2

Médias e desvios padrões sobre os construtos.

\begin{tabular}{c|c|c|c|c}
\hline Cód. & Construto & Média & Mediana & Desvio Padrão \\
\hline SP & $\begin{array}{c}\text { Similaridade } \\
\text { Percebida }\end{array}$ & 3,01 & 3,25 & 1,18 \\
\hline PC & $\begin{array}{c}\text { Percepção de } \\
\text { Crowding }\end{array}$ & 3,3 & 3,33 & 1,28 \\
\hline IC & Intenção de compra & 2,33 & 2,2 & 1,26 \\
\hline IL & Imagem de loja & 3,08 & 3,2 & 0,99 \\
\hline
\end{tabular}

Fonte: Elaboração dos autores.

De acordo com os dados da Tabela 2, verifica-se a partir das medidas de tendência centrais, média e mediana, a existência de similaridade entre os respondentes e as imagens utilizadas nos testes, além disso, o desvio padrão nas respostas dos sujeitos da pesquisa $(1,18)$ demonstram baixa dispersão entre as respostas. 0 mesmo ocorre com a percepção de crowding sendo essa a que obteve maior média $(3,3)$.

Quanto a intenção de compra por parte dos sujeitos da pesquisa verificou-se uma baixa intenção de compra, essa recebeu a menor média $(2,33)$. No entanto, a imagem de loja foi avaliada positivamente entre os participantes, apresentando a segunda maior média $(3,08)$ e o menor desvio padrão $(0,99)$.

\section{Validação do experimento}

Considerando a natureza dos experimentos, pode-se afirmar que o modelo apresentado é eficiente para descrever as relações existentes entre as diferentes variáveis: 
a) Verificar se o efeito da densidade foi alcançado

No que se refere à verificação do efeito da densidade, pode-se aferir que o experimento é capaz de representar os dados medidos e suas medidas experimentais flutuam em torno dos valores previstos $(p$-valor $<0,05)$, apresentado na Quadro 3.

\begin{tabular}{|c|c|c|c|c|c|c|c|}
\hline \multirow{3}{*}{ Densidade } & \multicolumn{3}{|c|}{ Alta } & \multicolumn{2}{c|}{ Baixa } & & \\
\cline { 2 - 8 } & Média & DP & Média & DP & T & DF & $p$ \\
\cline { 2 - 8 } & 2,99 & 1,25 & 1,8 & 0,99 & 5,9492 & 113,51 & 0,00001 \\
\hline
\end{tabular}

Fonte: Elaboração dos autores.

Quadro 3: Efeito da densidade.

Assim, infere-se que o efeito da densidade foi alcançado, sendo perfeitamente capaz de descrever as relações existentes entre as variáveis do problema, explicitando como as variáveis dependentes respondem a mudanças das condições experimentais e dos parâmetros.

b) Verificar se o efeito do perfil de outros consumidores foi alcançado

De maneira análoga à verificação do efeito da densidade, os valores previstos no que se refere ao perfil (p-valor < 0,05), são apresentados na Quadro 4.

\begin{tabular}{|c|c|c|c|c|c|c|c|}
\hline \multirow{3}{*}{ Efeito } & \multicolumn{2}{|c|}{ Perfil A } & \multicolumn{2}{c|}{ Perfil B } & \multicolumn{3}{c|}{} \\
\cline { 2 - 8 } & Média & DP & Média & DP & T & DF & $p$ \\
\cline { 2 - 8 } & 3,26 & 1,18 & 2,8 & 1,15 & 2,2595 & 125,69 & 0,02557 \\
\hline
\end{tabular}

Fonte: Elaboração dos autores.

Quadro 4: Perfil de outros consumidores.

O que demonstra que, o efeito do perfil de outros consumidores foi alcançado.

\section{Achados empíricos}

A primeira hipótese prediz que crowding está relacionado com a imagem negativa (positiva) da loja. Por meio dos resultados essa hipótese é suportada, onde no Quadro 3, podemos observar um $p$ value $=0,02164$, demonstrando a influência da densidade humana na percepção do consumidor com relação a imagem de loja. Esse resultado corrobora com os estudos de Lee, Kim e Li (2011), que relata sobre a existência de uma correlação positiva entre crowding e a influência sob percepção da imagem da loja.

Esses resultados confirmam os estudos de Eroglu e Harrell (1986); Boyko e Cooper (2011); Oliveira, Silva, Brandão e Lopes (2017) e Aguiar e Farias (2020), onde a percepção positiva sobre a imagem de loja está atrelada ao contexto de promoções na loja com desconto de produtos, ou seja, uma grande aglomeração de pessoas pode indicar oportunidade de compra mais barata.

A segunda hipótese propunha que crowding gera um menor (maior) intenção de compra. Porém, os dados não sustentam a hipótese, no Quadro 3 podemos observar um $p$-value $=0,1137$. Esse resultado evidencia que a densidade humana presente não influencia na decisão de compra do consumidor jovem.

Isto permite supor que por mais que os jovens universitários possam ver aglomeração de pessoas como algo positivo (Lee et al., 2011; Oliveira, Silva, Brandão, \& Lopes, 2017), ela por si só, não é determinante para decisão de compra do consumidor. As emoções, por exemplo, têm um papel 
moderador significativo no comportamento do consumidor jovem, como afirma os estudos de Baker e Wakefield (2012).

Quanto às $\mathrm{H} 2 \mathrm{a}$ e H2B, foi evidenciado que o perfil do consumidor não necessariamente influencia os sujeitos na percepção da imagem de loja, nem na sua intenção de compra, embora estudos como o de Ceretta e Froemming (2011) evidenciam que o público jovem tem tendência a se preocupar com sua aparência, sobretudo quando estão em locais públicos.

Dos quatro constructos, a Imagem da Loja é a que apresenta os melhores resultados, com coeficiente de variação de $32 \%$, a menor entre os quatro, indicando assim menor dispersão das respostas. Isso evidencia que para o público jovem a presença social tem maior efeito sobre a imagem que ele terá sobre a loja.

Dessa forma, se esperaria que a diferença de perfil dos consumidores, caracterizado pela alteração das vestimentas, representasse alguma mudança significativa na imagem do ambiente varejista e na sua intenção de compra. Porém, estas hipóteses foram refutadas o que pode ser observado no Quadro 5, sendo uma surpresa, visto que, os jovens, segundo os estudos de Thakor et al. (2008) são facilmente influenciáveis pelo perfil de outros consumidores.

\begin{tabular}{|c|c|c|c|c|c|c|}
\hline Hipótese & Previsão & Relacionamento & Teste T & $p$-value & Médias & $\begin{array}{c}\text { Resultado da } \\
\text { hipótese }\end{array}$ \\
\hline H1a & $\begin{array}{c}\text { Positiva } \\
\text { ou } \\
\text { negativa }\end{array}$ & $\begin{array}{c}\text { Densidade humana/ } \\
\text { imagem de loja }\end{array}$ & $-2,3249$ & 0,02164 & $\begin{array}{c}\text { Alta (2,88)/ } \\
\text { Baixa (3,25) }\end{array}$ & Suportada \\
\hline H1b & $\begin{array}{c}\text { Maior ou } \\
\text { menor }\end{array}$ & $\begin{array}{c}\text { Densidade humana/ } \\
\text { intenção de compra }\end{array}$ & $-1,5926$ & 0,1137 & $\begin{array}{c}\text { Alta (3,14)/ } \\
\text { Baixa (3,44) }\end{array}$ & Nuportada \\
\hline H2a & $\begin{array}{c}\text { Diferença } \\
\text { consumidor/ } \\
\text { imagem de loja }\end{array}$ & $-1,4377$ & 0,153 & $\begin{array}{c}\text { Perfil A (3,20)/ } \\
\text { Perfil B (2,97) }\end{array}$ & $\begin{array}{c}\text { Suportada } \\
\text { Suportada }\end{array}$ \\
\hline H2b & Diferença & $\begin{array}{c}\text { Perfil do } \\
\text { consumidor/ } \\
\text { intenção de compra }\end{array}$ & $-1,1812$ & 0,2397 & $\begin{array}{c}\text { Perfil A (3,42)/ } \\
\text { Perfil B (3,20) }\end{array}$ & Suportada \\
\hline
\end{tabular}

Fonte: Elaboração dos autores.

Quadro 5: Resumo dos resultados das hipóteses.

Por fim, estes resultados demonstram a imprevisibilidade decorrente do público jovem quanto ao seu perfil e comportamento no ambiente de varejo, isto pode ser motivado pelas constantes influências que esse público está suscetível e que podem moldar o seu perfil.

\section{CONCLUSÕES}

Durante todo percurso da pesquisa pode-se dizer que o objetivo geral foi alcançado, onde, através de análises estatísticas, foi possível verificar de que maneira a densidade humana e o perfil dos outros consumidores influenciam a imagem do varejista e as intenções de compra do consumidor jovem. Como resultado, foi demonstrado que a densidade humana pode influenciar a imagem de loja, entretanto, ela por si só não necessariamente influencia a decisão de compra dos sujeitos da pesquisa.

No que se refere ao perfil dos outros consumidores, nem a imagem de loja e nem intenção de compra são significativamente afetadas por essa variável. Isso foi algo que contraria o senso comum de que o público jovem é mais influenciável pelo perfil dos outros. Dessa forma, era esperado que a alteração do perfil do consumidor afetasse a imagem do ambiente varejista.

0 estudo também vem a contribuir teoricamente, quanto ao fenômeno de crowding no comportamento do consumidor no ambiente de varejo, assim como, estudos anteriores de Brandão e Parente (2013), Ferreira, Bizarrias, Silva e Brandão (2015), Aguiar e Farias (2015), Oliveira et al. 
(2017) e Aguiar e Farias (2020), entre outros. A pesquisa contribuiu na compreensão das possíveis relações entre esse fenômeno e o comportamento consumidor de jovens universitários.

Para os gestores, os resultados encontrados colaboram na elaboração de suas estratégias de marketing e no controle espacial, em razão de entenderem que a densidade humana pode afetar a imagem varejista, possibilitando a melhora de aspectos como o número de filas e distribuição de seus produtos pelos corredores de loja, permitindo melhor controle sobre o fluxo de pessoas.

No entanto, existiram algumas limitações de pesquisa, como o fato desta não ter sida realizada em um contexto real, e sim realizada através de um survey com questionários online, o que foi um problema quanto ao tempo de pesquisa. Entretanto, mesmo esta não tendo sido em campo, não impediu que as manipulações das variáveis fossem validadas e que os resultados encontrados fossem condizentes com o objetivo geral. É importante ressaltar que os resultados encontrados no presente artigo não podem ser generalizados, por se tratar de sujeitos de pesquisa bastante específicos.

Como sugestão de pesquisas futuras, a replicação do estudo modificando os sujeitos da pesquisa para pessoas de idade mais avançada. Através dessa modificação, se busca entender melhor a percepção desses novos sujeitos de estudo em relação a densidade humana e o perfil dos outros consumidores, buscando evidenciar semelhanças ou diferenças entre a percepção do público mais jovem, bem como a realização desta em campo, o que pode confirmar ou contradizer os resultados obtidos de acordo com as circunstâncias apresentadas. Ainda como sugestão para pesquisas futuras, o direcionamento a partir deste estudo para análises da influência do fenômeno de Crowding na intenção de compra em tempos de pandemia do Covid-19.

\section{Referências}

Aguiar, E. C. (2016). O Papel Moderador da Similaridade Percebida na Relação entre Percepção de Crowding e Respostas do Consumidor em Ambiente Varejista (Tese de doutorado). Recife, PE, Universidade Federal de Pernambuco.

Aguiar, E. C., \& Farias, S. A. (2020). Identificar-se com os outros consumidores minimiza o efeito crowding? 0 papel da similaridade percebida. Revista Eletrônica de Ciência Administrativa, 19(1), 083-103.

Aguiar, E. C., \& Farias, S. A. (2015). Percepção da presença dos outros consumidores e sua relação com emoções e valor hedônico de compra. Revista de Administração de Empresas, 55(6), 712-723.

Aguiar, E. C., Farias, S. A., Gomes, V. M. S., \& Gomes, J. G. (2015). Percepção de crowding e comportamento do consumidor: uma abordagem não linear no varejo supermercadista de baixa renda. Revista de Administração da UNIMEP, 13(3), 207-229.

Albuquerque, M. P. C. (2007). Análise da evolução do setor supermercadista brasileiro: Uma visão estratégica (Dissertação de Mestrado). Rio de Janeiro, RJ, Faculdade de Economia e Finanças IBMEC.

Baker, J. (1986). The Role of the Environment in Marketing Services: The Consumer Perspective. The Services Challenge: Integrating for Competitive Advantage, 1(1), 79-84.

Baker, J., \& Wakefield, K. L. (2012). How consumer shopping orientation influences perceived crowding, excitement, and stress at the mall. Journal of the Academy of Marketing Science, 40(6), 791-806.

Barki, E., Botelho, D., \& Parente, J. G. (2013). Varejo desafio e oportunidades nos em mercados emergentes. Revista de Administração de Empresas, 53(6), 534-538.

Boyko, C., \& Cooper, R. (2011). Clarifying and Re-conceptualising Density. Progress in Planning, 76(1), $1-61$.

Brandão, M. M., \& Parente, J. G. (2013). Brasileiro gosta de muvuca? Impacto da densidade humana no comportamento de compra. Revista de Administração de Empresas, 52(6), 613-627.

Brandão, M. M. (2012). Crowding no varejo: diferenças na satisfação e percepção de valor hedônico de consumidores de alta e baixa renda no Brasil (Tese de doutorado). São Paulo, SP, Escola de Administração de Empresas de São Paulo, FGV/EAESP. 
Brocato, E. D., Voorhees, C. M., \& Baker, J. (2012). Understanding the influence of cues from other customers in the service experience: a scale development and validation. Journal of Retailing, 88(3), 384-398.

Byrne, D. E. (1971). The attraction paradigm (11 a ed.). New York: Academic Press.

Ceretta, S. B., \& Froemming, L. M. (2011). Geração Z: compreendendo os hábitos de consumo da geração emergente. Revista Eletrônica do Mestrado Profissional em Administração da Universidade Potiguar, 3(2), 15-24.

Eroglu, S. A., \& Harrell, G. D. (1986). Retail Crowding: Theoretical and Strategic Implications. Journal of Retailing, 62(4), 346-363.

Eroglu, S. A., \& Machleit, K. A. (1990). An empirical study of retail crowding: antecedents and consequences. Journal of Retailing, 66(2), 201-221.

Eroglu, S. A., Machleit, K. G. D., \& Barr, T. F. (2005). Perceived Retail Crowding and Shopping Satisfaction: The Role of Shopping Calues. Journal of Business Research, 58(8), 1146-1153.

Ferreira, M. C. O., Bizarrias, F. S., Silva, J. G., \& Brandão, M. M. (2015). Muvuca na Loja Aumenta Compra por Impulso ou Afasta Consumidores? A Resposta Depende da Tolerância ao Crowding. Revista Brasileira de Marketing, 14(4), 545-556.

Gil, A. C. (2002). Como elaborar projetos de pesquisa (4⿳亠丷a ed.). São Paulo: Atlas.

Golden, L. L., Albaum, G., \& Zimmer, M. (1987). The Numerical Comparative Scale: An economical format for R. Journal of Retailing, 63(4), 393.

Grewal, D., Baker, J., Levy, M., \& Voss, G. B. (2003). The effects of wait expectations and store atmosphere evaluations on patronage intentions in service-intensive retail stores. Journal of Retailing, 79(4), 259-268.

Hair, J. F., Jr., Black, W. C., Babin, B. J., Anderson, R. E., \& Tatham, R. L. (2009). Análise multivariada de dados (6 $\mathrm{a}$ ed.). Porto Alegre: Bookman Editora.

Hui, M. K., \& Bateson, J. E. G. (1991). Perceived Control and the Effects of Crowding and Consumer Choice on the Service Experience. Journal Consumer Research, 18(2), 174-184.

Kukar-Kinney, M., \& Grewal, D. (2007). Comparison of consumer reactions to price-matching guarantees in internet and bricks-and-mortar retail environments. Journal of the Academy of Marketing Science, 35(2), 197-207.

Kukar-Kinney, M., Walters, R. G., \& Mackenzie, S. B. (2007). Consumer responses to characteristics of price-matching guarantees: The moderating role of price consciousness. Journal of Retailing, 83(2), 211-221.

Kwon, H., Ha, S., \& Im, H. (2016). The impact of perceived similarity to other customers on shopping mall satisfaction. Journal of Retailing and Consumer Services, 28(1), 304-309.

Latané, B. (1981). The psychology of social impact. American Psychologist, 36(4), 343-356.

Lee, S. Y., Kim, J. O., \& Li, J. G. (2011). Impacts of store crowding on shopping behavior and store image. Journal of Asian Architecture and Building Engineering, 10(1), 133-140.

Machleit, K. A., Eroglu., S. A., \& Mantel, S, P. (2000). Perceived retail crowding and shopping satisfaction: what modifies this relationship? Journal of Consumer Psychology, 9(1), 29-42.

Machleit, K. A; Kellaris, J. J., \& Eroglu, S. A. (1994). Human versus spatial dimensions of crowding perceptions in retail environments: a note on their measurement and effect on shopper satisfaction. Marketing Letters, 5(1), 183-194.

Malhotra, N. K. (2012). Pesquisa de Marketing: Uma Orientação Aplicada (6 $\underline{\mathrm{a}}$ ed.). Porto Alegre: Bookman Editora.

Mehrabian, A., \& Russell, J. (1974). An approach to environmental psychology (1o ed.). Cambridge: MIT Press.

Oliveira, A. S., Silva, D., Brandão, M. M., \& Lopes, E. L. (2017). Influência do crowding na lealdade mediado pela satisfação do consumidor em processos de compras no varejo. Revista de Administração da Universidade Federal de Santa Maria, 10(4), 614-631.

Peck, J., \& Childers, T. L. (2003). To have and to hold: The influence of haptic information on product judgments. Journal of Marketing, 67(2), 35-48. 
Raajpoot, N., Jackson, A., \& Lefebvre, J. (2013). Non-verbal customer-to-customer interaction in retail setting: An investigation of indirect effects of perceived customer similarity on important marketing outcomes. Atlantic Marketing Journal, 2(1), 16-41.

Rompay, T. J. L. van, Galetzka, M., Pruyn, A. T. H., \& Garcia, J. M. (2008). Human and spatial dimensions of retail density: revisiting the role of perceived control. Psychology \& Marketing, 25(4), 319-335.

Santos, G. G., \& Santos, A. G. (2019). Administração e Marketing no Varejo: Conceitos e práticas no mercado (1aㅡ ed). São Paulo: Edição do Kindle.

Silveira, N. S. P., \& Hanashiro, D. M. M. (2009). Similaridade e dissimilaridade entre superiores e subordinados e suas as implicações para a qualidade da relação diádica. Revista de Administração Contemporânea, 13(1), 117-135.

Sirgy, M. J., Grewal, D., Mangleburg, T. F., Park, J., Chon, K., Claiborne, C. B., Johar, J. S., Berkman, H. (1997). Assessing the predictive validity of two methods of measuring self-image congruence. Journal of the Academy of Marketing Science, 25(3), 229-241.

Srivastava, J., \& Lurie, N. H. (2004). Price-matching guarantees as signals of low store prices: survey and experimental evidence. Journal of Retailing, 80(2), 117-128.

Stokols, D. (1972). On the Distinction Between Density and Crowding: Some Implications for Future Research. Psychological Review, 79(3), 275-278.

Thakor, M. V., Suri, R., \& Saleh, K. (2008). Effects of service setting and other consumers: age on the service perceptions of young consumers. Journal of Retailing, 84(2), 137-149.

Uhrich, S., \& Benkenstein, M. (2012). Physical and social atmospheric effects in hedonic service consumption: Customers' roles at sporting events. The Service Industries Journal, 32(11), 17411757.

Wickens, T. D., \& Keppel, G. (2004). Design and analysis: A researcher's handbook (4ํㅜ ed.). Upper Saddle River: Pearson Prentice-Hall.

Apêndice A - Descrição do contexto de cada experimento

Condição 1: Você está passeando em um shopping da sua cidade quando você vê uma loja de roupas que atrai sua atenção. Você entra na loja para olhar os produtos. Você percebe que também há outros clientes na loja (Imagem com clientes com perfil A). Agora, por favor, releia o parágrafo, olhe para a imagem de compra com outros clientes (imagem com clientes de perfil A e Densidade Humana alta). Depois responda cada uma das perguntas tendo em mente que você está neste ambiente de compra.

Condição 2: Você está passeando em um shopping da sua cidade quando você vê uma loja de roupas que atrai sua atenção. Você entra na loja para olhar os produtos. Você percebe que também há outros clientes na loja (Imagem com clientes com perfil A). Agora, por favor, releia o parágrafo, olhe para a imagem de compra com outros clientes (imagem com clientes de perfil A e Densidade Humana baixa). Depois responda cada uma das perguntas tendo em mente que você está neste ambiente de compra.
Condição 3: Você está passeando em um shopping da sua cidade quando você vê uma loja de roupas que atrai sua atenção. Você entra na loja para olhar os produtos. Você percebe que também há outros clientes na loja (Imagem com clientes com perfil B). Agora, por favor, releia o parágrafo, olhe para a imagem de compra com outros clientes (imagem com clientes de perfil $B$ e Densidade Humana alta). Depois responda cada uma das perguntas tendo em mente que você está neste ambiente de compra.

Condição 4: Você está passeando em um shopping da sua cidade quando você vê uma loja de roupas que atrai sua atenção. Você entra na loja para olhar os produtos. Você percebe que também há outros clientes na loja (Imagem com clientes com perfil B). Agora, por favor, releia o parágrafo, olhe para a imagem de compra com outros clientes (imagem com clientes de perfil B e Densidade Humana baixa). Depois responda cada uma das perguntas tendo em mente que você está neste ambiente de compra.

Fonte: Elaboração dos autores. 
Apêndice B - Escalas utilizadas

\begin{tabular}{|c|c|}
\hline Construtos & Escalas \\
\hline \multirow{4}{*}{$\begin{array}{l}\text { Similaridade percebida (Byrne, 1971; Sirgy et al., } \\
\text { 1997; Brocato et al., 2012) }\end{array}$} & "Eu me identifico com os clientes desta loja" \\
\hline & "Eu sou parecido com os outros clientes desta loja" \\
\hline & "Os clientes desta loja são similares a mim" \\
\hline & "Eu combino com os outros clientes desta loja". \\
\hline \multirow{2}{*}{$\begin{array}{l}\text { Percepção de Crowding (Eroglu \& Machleit, 1990; } \\
\text { Machleit, Kellaris, \& Eroglu, 1994) }\end{array}$} & "Esta loja parece estar muito cheia para mim" \\
\hline & "Na minha opinião, tem muito movimento nesta loja". \\
\hline \multirow{3}{*}{$\begin{array}{l}\text { Intenção de compra (Mehrabian \& Russell, 1974; } \\
\text { Grewal et al., 2003; Rompay, Galetzka, Pruyn, \& Garcia, } \\
\text { 2008) }\end{array}$} & "Eu consideraria fazer compras nesta loja" \\
\hline & "Eu estaria disposto a fazer compras nesta loja" \\
\hline & $\begin{array}{l}\text { "Muito provavelmente eu realizaria compras nesta } \\
\text { loja" }\end{array}$ \\
\hline \multirow{3}{*}{$\begin{array}{c}\text { Percepção de preço (Srivastava \& Lurie, 2004; Kukar- } \\
\text { Kinney \& Grewal, 2007, Kukar-Kinney, Walters, \& } \\
\text { Mackenzie, 2007) }\end{array}$} & $\begin{array}{c}\text { "Eu acho que os preços praticados nesta loja são } \\
\text { adequados" }\end{array}$ \\
\hline & $\begin{array}{l}\text { "Na minha opinião os preços dos produtos desta loja } \\
\text { estão dentro da média" }\end{array}$ \\
\hline & $\begin{array}{c}\text { "Na minha opinião os preços dos produtos desta loja } \\
\text { estão dentro da média do setor (outras lojas do } \\
\text { mesmo segmento)" }\end{array}$ \\
\hline \multirow{4}{*}{$\begin{array}{c}\text { Imagem de loja: (Golden, Albaum, \& Zimmer, 1987; } \\
\text { Grewal, Baker, Levy, \& Voss, 2003) }\end{array}$} & "Essa loja me parece ser: Uma boa loja" \\
\hline & "Essa loja me parece ser: Uma loja agradável" \\
\hline & "Essa loja me parece ser: Uma loja de qualidade" \\
\hline & "Essa loja me parece ser: Uma loja arrumada" \\
\hline \multirow{3}{*}{$\begin{array}{l}\text { Comportamento de aproximação e afastamento } \\
\text { (Mehrabian \& Russell, 1974; Grewal et al., 2003) }\end{array}$} & "Eu gostaria de andar por esta loja" \\
\hline & "Eu gostaria de permanecer nesta loja" \\
\hline & $\begin{array}{l}\text { "Eu gostaria de passar um tempo andando por esta } \\
\text { loja" }\end{array}$ \\
\hline \multirow{3}{*}{ Tolerância ao Crowding (Machleit et al., 2000) } & "Sempre que possível, evito lojas lotadas" \\
\hline & "Eu não me incomodo com uma loja lotada" \\
\hline & "Eu não entro numa loja quando ela está lotada" \\
\hline \multirow{4}{*}{ Necessidade de toque (Peck \& Childers, 2003) } & $\begin{array}{c}\text { "Eu confio mais nos produtos que podem ser tocados } \\
\text { antes da compra" }\end{array}$ \\
\hline & $\begin{array}{l}\text { "Me sinto mais confortável comprando um produto } \\
\text { depois de examiná-lo fisicamente" }\end{array}$ \\
\hline & $\begin{array}{l}\text { "Se não consigo tocar no produto da loja, fico com } \\
\text { receio de comprar o produto" }\end{array}$ \\
\hline & $\begin{array}{l}\text { "Eu me sinto mais confiante de comprar o produto } \\
\text { depois de tocá-lo" }\end{array}$ \\
\hline
\end{tabular}

Anexo A - Cenários (fotografias) utilizados no experimento - Condições do experimento

Perfil A, Densidade Humana Alta

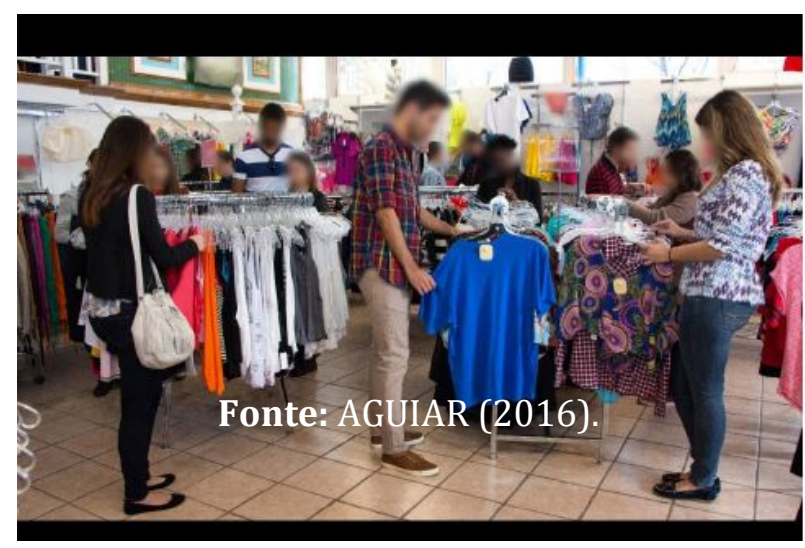

Perfil A, Densidade Humana Baixa

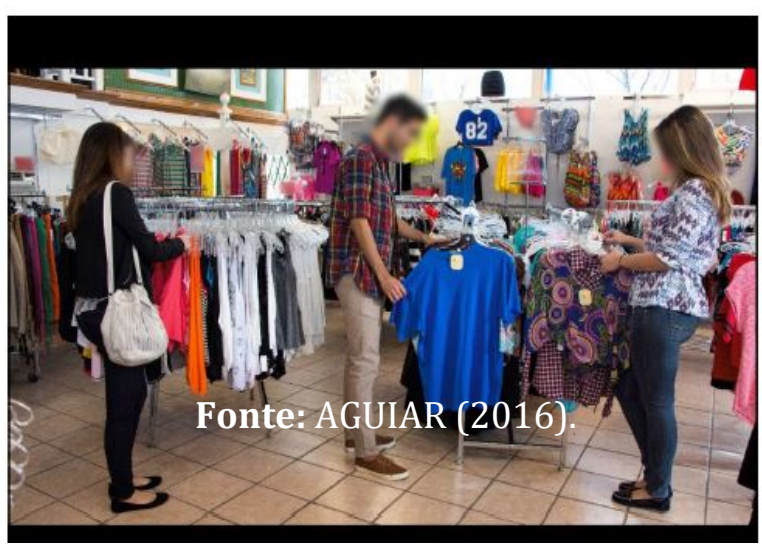


Perfil B, Densidade Humana Alta.

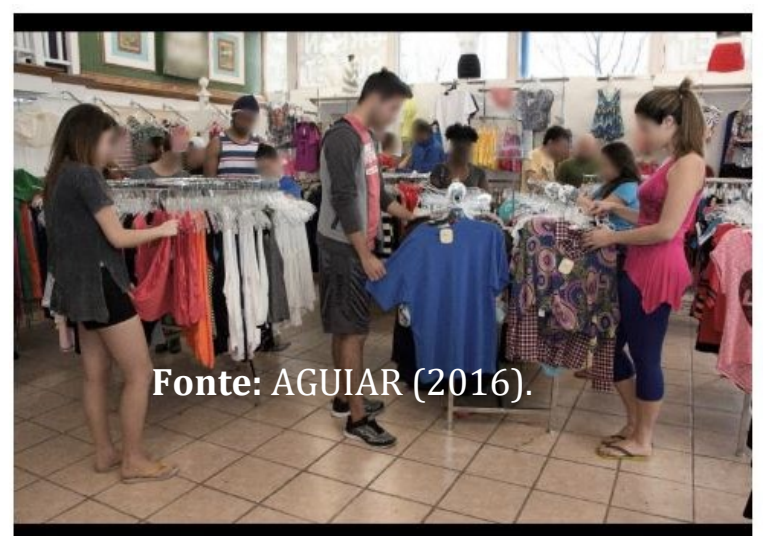

Perfil B, Densidade Humana Baixa.

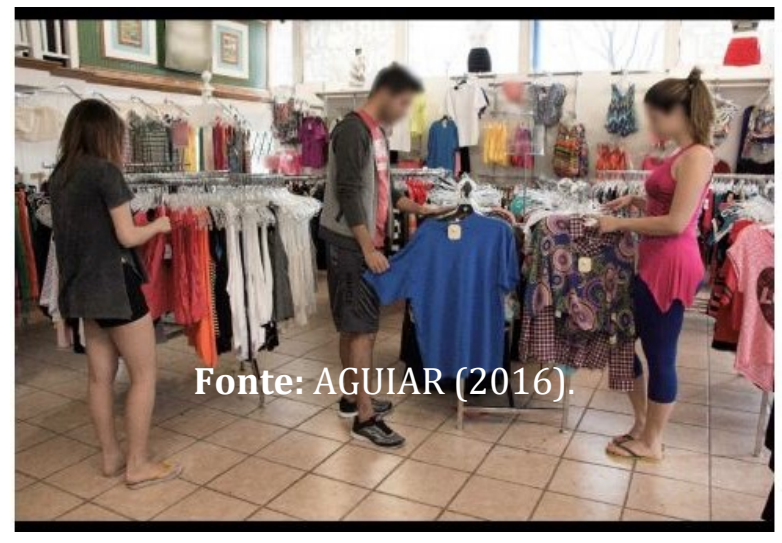

